\section{In-vitro retinal model reveals a sharp transition between laser damage mechanisms}

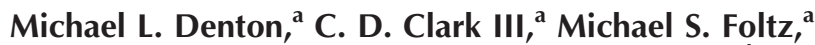 \\ Kurt J. Schuster, a Gary D. Noojin, ${ }^{\text {a Larry E. Estlack, }}$ \\ and Robert J. Thomas ${ }^{c}$ \\ ${ }^{a}$ TASC, Inc., Biomedical Sciences and Technologies \\ Department, Brooks City-Base, Texas 78235 \\ ${ }^{\mathrm{b} C o n c e p t u a l}$ MindWorks, Inc., San Antonio, Texas 78228 \\ 'Air Force Research Laboratory, 711 HPW/RHDO, \\ Brooks City-Base, Texas 78235
}

\begin{abstract}
We use laser damage thresholds in an in-vitro retinal model, and computational simulations to examine the laser exposure durations at which damage transitions from photothermal to photochemical at $413 \mathrm{~nm}$. Our results indicate a dramatic shift in 1-h damage thresholds between exposure durations of 60 and $100 \mathrm{~s}$. The trend in our in-vitro results is similar to a trend found in a recent study where retinal lesions were assessed 1-h post laser exposure in the rhesus eye Our data suggest that nonthermal mechanisms did not significantly contribute to cell death, even for exposures of $60 \mathrm{~s}$. Knowledge of the transition point, and lack of concurrent thermal and nonthermal damage processes, are significant for those wishing to devise a comprehensive computational damage model. (1) 2010 Society of Photo-Optical Instrumentation Engineers. [DOI: $10.1117 / 1.3449107]$
\end{abstract}

Keywords: laser-induced damage; cells; thermal effects; nonthermal effects.

Paper 10136LR received Mar. 18, 2010; revised manuscript received May 7, 2010; accepted for publication May 7, 2010; published online Jun. 14, 2010.

Cells exposed to laser pulses longer than the thermal diffusion rate can experience damage by either photothermal or photochemical mechanisms, depending on wavelength, exposure duration, and beam diameter. In addition, laser irradiance (E) dictates the degree of chromophore-dependent heating in tissues. In general, the irradiance requirement for damage is reduced as exposure duration is extended. This trend explains why wavelength-dependent nonthermal damage only predominates for long laser exposures, and how thermal damage can be forced to occur from relatively short exposures. Radiant exposure $(\mathrm{H})$ is used to correlate laser dose to damage outcomes, and is calculated by multiplying irradiance $\left(\mathrm{W} \mathrm{cm}^{-2}\right)$ by exposure duration.

In animals, where measurements of temperature rise and oxidative products are difficult, researchers have relied on evaluations of trends in damage thresholds relative to wavelength (action spectrum) and exposure duration [temporal action profile (TAP) $]^{1}$ to distinguish a damage mechanism. The principal of irradiance reciprocity, where threshold irradiance

Address all correspondence to: Robert J. Thomas, E-mail: robert.thomas@brooks.af.mil is inversely proportional to exposure duration, is a prominent feature of photochemical (nonthermal) damage, and is easily identified in TAP analyses using threshold irradiance (ETAP) or threshold radiant exposure (HTAP). Ocular lesions assessed at 24-h post exposure (or longer) for exposures of $100 \mathrm{~s}$ (or longer) at $514 \mathrm{~nm}$ (or shorter) were considered photochemical. ${ }^{1,2}$ When lesions from 514-nm exposures were assessed at $1 \mathrm{~h}$, damage from a 1000-s exposure was consistent (HTAP) with photothermal processes. ${ }^{2}$ Therefore, this "latency" in the appearance of damage is also useful in characterizing laser damage mechanisms.

While characterizing a novel in-vitro retinal model, ${ }^{3}$ we made note of a peculiar trend for damage thresholds at $413 \mathrm{~nm}$ that compared with in-vivo thresholds at 457.9 and $441.6 \mathrm{~nm}$ reported by Lund, Stuck, and Edsall. ${ }^{4}$ An HTAP analysis of the data revealed thresholds that continued along the thermal trend line to threshold radiant exposure values greater than that for $100 \mathrm{~s}$, which was expected to be at irradiance reciprocity. Figure 1(a) is an HTAP for the 441.6-nm (327- $\mu \mathrm{m}$ beam diameter) data taken from Ref. 4 . Without the usual log-log axis, the HTAP clearly shows that the 1-h post exposure threshold values for the 5-, 8-, and 16-s exposures are greater than that of the photochemical threshold $\left(26 \mathrm{~J} \mathrm{~cm}^{-2}\right)$. As the authors concluded, ${ }^{4}$ a lack of latent lesions following laser exposures of $5 \mathrm{~s}$ and shorter indicated damage by thermal mechanisms. However, the fact that threshold values for the 8- to 16-s exposures did not decrease to the level of the 100-s threshold implied the possibility of mixed damage mechanisms in the progression to purely photochemical processes. The authors discussed how the 16-s threshold data suggested a gradual transition between mechanisms due to a delayed appearance of threshold lesions (indicating nonthermal damage) and a dependence on the diameter of the irradiated retinal area (indicating thermal damage).

In this study, we use a combination of new in-vitro damage data and computational simulations of both temperature rise and damage thresholds at $413 \mathrm{~nm}$ to address the shift to photochemical damage processes in more detail. Except for the following changes, laser exposures were as described previously. ${ }^{3}$ Retinal pigment epithelial (RPE) cells (about 160 melanosomes/cell) were exposed to 413-nm laser irradiation in 48-well plates containing $0.1-\mathrm{mL}$ complete Hank's balanced salt solution. Cells were exposed to a $0.3-\mathrm{mm}$-diam flat-top beam (via an 88-mm focal length lens) in an environmentally controlled enclosure (Fig. 2) that ensured consistent temperature $\left(35\right.$ to $37^{\circ} \mathrm{C}$ ) and relative humidity (60 to $\left.70 \%\right)$. Estimated dose for $50 \%$ lethality $\left(\mathrm{ED}_{50}\right)$ values were calculated using the Probit method, ${ }^{5,6}$ where Probit slopes represent the first derivative with respect to dose at a probability of 0.5 . Systematic uncertainty $(15 \%)$ in our irradiance values was calculated as previously described. ${ }^{7}$

To simulate the experimental exposures, we used a lasertissue damage program developed by our group. ${ }^{8}$ The model uses a laser propagation model (geometric optics assumed) to compute a source term (implementing Beer's law of linear absorption) for a thermal heat solver. The heat solver accounts for the multiple layers comprising the system (buffer, cells, and plastic well plate) and air/surface boundary conditions to predict temperature rise at the 7 - $\mu$ m-thick cell layer. These 
(a)

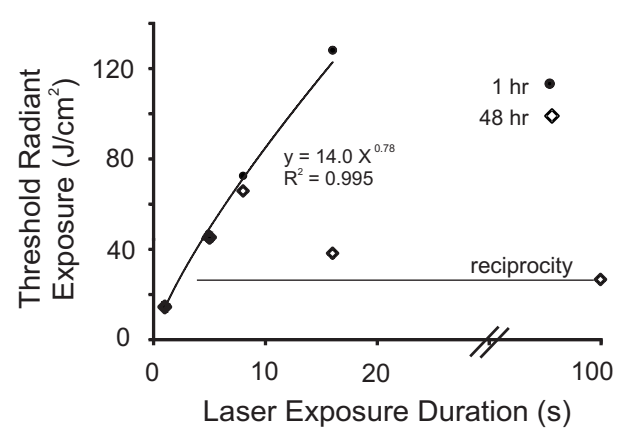

(b)

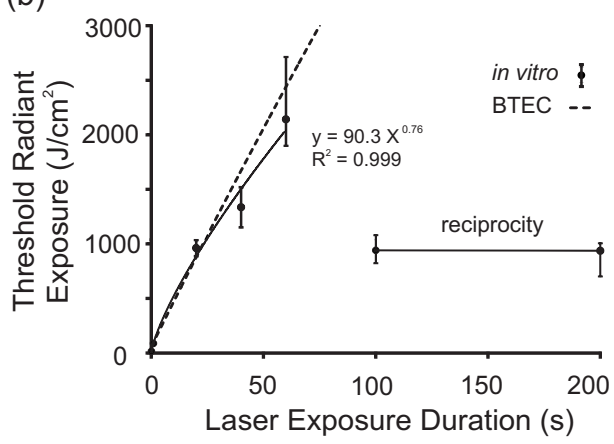

Fig. 1 Comparison of in-vivo and in-vitro damage trends. (a) HTAP for 1- and 48-h damage thresholds (441.6 nm) in the in-vivo MVL study of Ref. 4. The 100-s 48-h data point is expected to represent irradiance reciprocity. (b) HTAP for 1-h damage thresholds (413 nm) in the in-vitro retinal model. Estimated thresholds using the BTEC thermal model are represented by the dashed line. Error bars depict upper and lower fiducial limits ( 95 $\%$ confidence intervals).

temperatures were input into an Arrhenius rate equation that was numerically integrated to determine damage outcomes. The two Arrhenius rate parameters $\left(A=3.1 \times 10^{99} \mathrm{~s}^{-1} ; E_{a}\right.$ $\left.=6.28 \times 10^{5} \mathrm{~J} / \mathrm{mol}^{-1}\right)$ used in the damage integral $(\Omega)$ calculation were those reported by Welch and Polhamus. ${ }^{9} \mathrm{~A}$ numerical search algorithm was used to determine the threshold irradiance that solved for an $\Omega$ value of 1 at the center of the beam.

The 1-h ED $\mathrm{ED}_{50}$ irradiances for the current in-vitro exposures are given in Table 1. Consistent environmental conditions during exposures led to low variance (fiducial limits) about the threshold values. Notice that, as expected, the irradiance requirement for damage was reduced by extending the duration of the laser exposure, although there was no significant change between the 40- and 60-s thresholds. Table 1 also shows that the threshold value for the 200-s exposure was exactly half the 100-s threshold, defining irradiance reciprocity (nonthermal damage) for the $0.3-\mathrm{mm}$ beam at $36{ }^{\circ} \mathrm{C}$ in the in-vitro retinal system.

An HTAP analysis of the in-vitro results [Fig. 1(b)] shows a trend similar to the 1-h assessments of Ref. 4 at $441.6 \mathrm{~nm}$ [Fig. 1(a)]. Power functions describing the in-vitro and in-vivo data had nearly identical exponents $(0.76$ and 0.78 , respec-

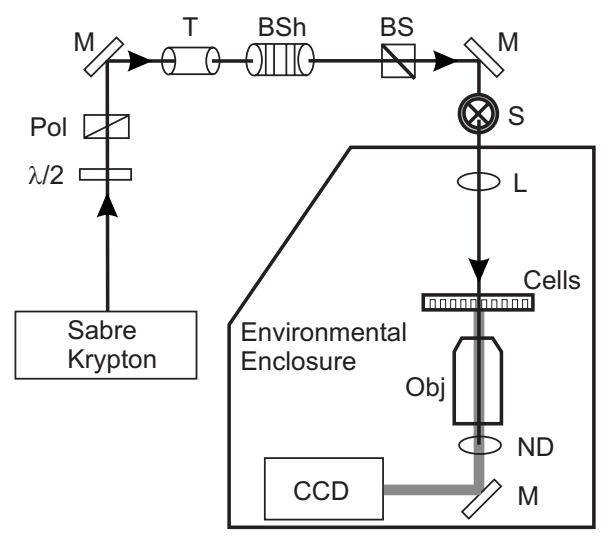

Fig. 2 Schematic representation of 413-nm beam delivery for in-vitro laser exposures. $M$, mirror; $\lambda / 2$, half-wave plate; Pol, polarizing beamsplitter; T, optical telescope; BSh, beam shaper; BS, beamsplitter; $S$, shutter; $L$, lens; ND, neutral density filter; $C C D$, charge-coupled device camera; and Obj, microscope objective. tively), but the in-vivo curve was shifted to lower threshold values by a factor of about 6.5 , presumably due to differences in melanosome density as discussed previously. ${ }^{3}$ Although difficult to see in Fig. 1(b), the threshold data points for the 0.1 - and 1.0-s exposures are near the origin. Notice the inverse relationship between irradiance and radiant exposure, such that the 0.1-s exposure required the greatest irradiance (Table 1) and the least radiant exposure [Fig. 1(b)] for generating threshold damage. However, as seen in Fig. 1, this trend is dramatically broken when the damage mechanism shifted to nonthermal, as indicated by irradiance reciprocity. This necessarily means that there was a significant thermal component in the death process for exposures of $1 \mathrm{~min}$. The correlation coefficient for the power function describing the 0.1 - to 60 -s data [see Fig. 1(b)] would suggest a similar degree of thermal component over this entire range of exposure durations.

As a means of understanding the thermal component of the in-vitro damage thresholds, we simulated irradiance threshold values $(\Omega=1)$ for exposure durations of 0.1 to $200 \mathrm{~s}$ and

Table 1 1-h damage threshold irradiance values for 413-nm laser exposures of in in-vitro retinal model. FL are Fiducial limits (95\% confidence interval). Systematic uncertainty was determined to be $15 \%$.

\begin{tabular}{cccccc} 
& & \multicolumn{5}{c}{$\begin{array}{c}\text { Threshold irradiance } \\
\left(\mathrm{W} \mathrm{cm}^{-2}\right)\end{array}$} \\
\cline { 3 - 6 } $\begin{array}{c}\text { Exposure } \\
\text { Duration } \\
(\mathrm{s})\end{array}$ & $\begin{array}{c}\text { Number of } \\
\text { samples }\end{array}$ & $\mathrm{ED}_{50}$ & $\begin{array}{c}\text { Lower } \\
\mathrm{FL}^{*}\end{array}$ & $\begin{array}{c}\text { Upper } \\
\mathrm{FL}\end{array}$ & $\begin{array}{c}\text { Probit } \\
\text { slope }\end{array}$ \\
\hline 0.1 & 94 & 157 & 145 & 169 & 15 \\
1.0 & 92 & 88.7 & 82.0 & 94.4 & 18 \\
20 & 71 & 48.1 & 41.3 & 51.8 & 18 \\
40 & 64 & 33.4 & 28.7 & 38.0 & 15 \\
60 & 66 & 35.7 & 31.6 & 45.2 & 9 \\
100 & 80 & 9.4 & 8.2 & 10.8 & 8 \\
200 & 24 & 4.7 & 3.5 & 5.0 & 24 \\
\hline
\end{tabular}




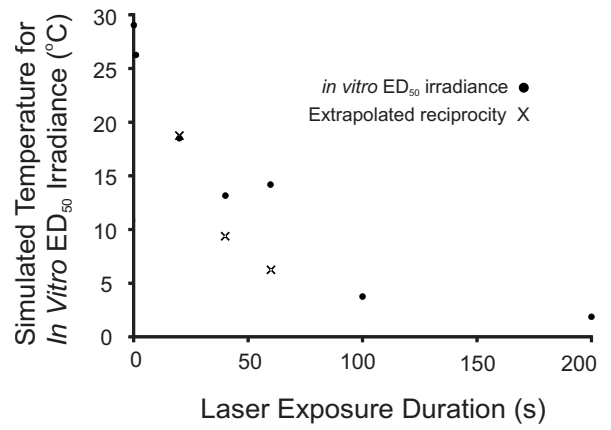

Fig. 3 Simulated (BTEC model) peak temperature rise values for invitro $\mathrm{ED}_{50}$ irradiance values (circles) as a function of exposure duration. Peak temperatures of irradiances extrapolated in accordance with reciprocity $\left(t^{-1}\right)$ were also simulated and shown.

plotted the radiant exposure results alongside the in-vitro data [Fig. 1(b)]. The simulated curve fits fairly well with the invitro data, falling within the $95 \%$ confidence intervals for all but the 40-s data point. The deviation in simulated and invitro HTAP curves suggests that mixed damage mechanisms may exist for exposures of 40 to $60 \mathrm{~s}$. However, we understand that our choice in values for the Arrhenius rate parameters, the fact that one of these parameters (frequency factor $A$ ) has a slight dependence on temperature, and our $15 \%$ systematic uncertainty (Table 1), are all factors that could lead to the observed disparity.

Looking at the irradiance thresholds in Table 1, we see no difference between the 40- and 60-s exposures, which means the continuation of radiant exposure thresholds along the power curve in the HTAP is directly proportional to the increase in exposure duration. However, this is not necessarily unexpected, because threshold irradiance (and temperature) is proportional to the inverse of log time under the Arrhenius damage model, which predicts a convergence of threshold irradiances (and temperatures) at longer exposure durations. A similar scenario appears to play out in the rhesus eye. The tabulated retinal 1 -h threshold irradiance values for the 5- to 16-s exposures reported in Ref. 4 were all three statistically identical as well.

Previous authors have used a peak temperature rise of 9 to $10^{\circ} \mathrm{C}$ to signify a minimum requirement for damage by thermal means. ${ }^{10}$ As expected, simulated peak temperature rises (Fig. 3) for the 100- and 200-s in-vitro threshold values were low (3.8 and $1.9^{\circ} \mathrm{C}$, respectively). Figure 3 also shows that simulated peak temperature rises of greater than $10^{\circ} \mathrm{C}$ were calculated for the in-vitro $\mathrm{ED}_{50}$ irradiance values for exposures of $60 \mathrm{~s}$ and shorter, which implies that sufficient heat was generated to produce death by thermal mechanisms. When we looked at the individual damage outcome data (damage versus no damage) for the 60-s exposures, we found that the lowest irradiance $\left(23.3 \mathrm{~W} \mathrm{~cm}^{-2}\right)$ that caused damage corresponded to a (simulated) temperature rise of $9.3^{\circ} \mathrm{C}$. Again, this indicated that all the $60-\mathrm{S}$ exposure damage outcomes had sufficient temperature rises to cause damage by thermal means. However, this does not exclude the possibility of nonthermal (photo-oxidation) events occurring concurrently with this elevation of temperature. The lack of an intermediate temperature rise for the 40 - and $60-\mathrm{S} \mathrm{ED}_{50}$ irradi- ance values, such as 5 to $9{ }^{\circ} \mathrm{C}$, suggests that if concurrent mechanisms did exist, they were neither additive nor synergistic to the overall damage rate process.

Finally, we were interested in the expected temperature rise of cells receiving the 40- and 60-s reciprocity irradiance doses. On extrapolating $\left(t^{-1}\right)$ from the 100- and 200-s irradiances, we simulated peak temperatures of 9.4 and $6.3{ }^{\circ} \mathrm{C}$ for the extrapolated irradiances corresponding to 40- and 60-s exposures, respectively. This suggests that if there did exist additive or synergistic effects from thermal and nonthermal damage mechanisms, it would be manifested in the data for 60-s exposures.

In conclusion, the in-vitro retinal model showed transitions in the damage mechanism for 1-h thresholds similar to those found previously in an in-vivo model. In the in-vitro model, the transition from photothermal to nonthermal damage was sudden, occurring somewhere between exposure durations of 60 and $100 \mathrm{~s}$. Additional data in this exposure range are being collected to characterize this transition. The current data cannot rule out the possibility of photochemical oxidation occurring during damaging 60-s exposures at $413 \mathrm{~nm}$. We are currently conducting experiments to address this issue. However, we believe that nonthermal processes do not contribute to cell death at $1-\mathrm{h}$ postexposure.

\section{Acknowledgments}

Any opinions, interpretations, conclusions, and recommendations are not necessarily endorsed by the U.S. Air Force. We thank C. Burney, B. Stuck, T. Khan, and D. Wooddell for helpful discussions regarding the manuscript. We would also like to thank H. Hodnett and D. Stolarski for technical assistance. This work was supported by the Air Force Research Laboratory (contract F41624-02-D-7003) and the Air Force Office of Scientific Research (grant 92HE04COR).

\section{References}

1. W. T. Ham Jr., J. J. Ruffolo Jr., H. A. Mueller, and D. Guerry III, "The nature of retinal radiation damage: Dependence on wavelength, power level and exposure time," Vision Res. 20, 1105-1111 (1980).

2. W. D. Gibbons and R. G. Allen, "Retinal damage from long-term exposure to laser radiation," Invest. Ophthalmol. Vis. Sci. 16, 521529 (1977).

3. M. L. Denton, M. S. Foltz, K. J. Schuster, G. D. Noojin, L. E. Estlack, and R. J. Thomas, "In vitro model that approximates retinal damage threshold trends," J. Biomed. Opt. 13(5), 054014 (2008).

4. D. J. Lund, B. E. Stuck, and P. Edsall, "Retinal injury threshold for blue wavelength lasers," Health Phys. 90(5), 477-484 (2006).

5. C. P. Cain, G. D. Noojin, and L. Manning, "A comparison of various probit methods for analyzing yes/no data on a log scale," USAF School of Aerospace Medicine, Brooks Air Force Base, TX, USAF Technical Report AL/OE-TR-1996-0102 (1996).

6. D. J. Finney, Probit Analysis, Cambridge University Press, New York (1971).

7. B. N. Taylor and C. E. Kuyatt, "Guidelines for evaluating and expressing the uncertainty of NIST measurement results," NIST Tech Note 1297 (1994).

8. L. Irvin, P. D. S. Maseberg, G. Buffington, C. D. Clark III, R. J. Thomas, M. L. Edwards, and J. Stolarski, "BTEC thermal model," USAF Technical Report AFRL-RH-BR-TR-2008-0006 (2008).

9. A. J. Welch and G. D. Polhamus, "Measurement and prediction of thermal injury in the retina of Rhesus monkey," IEEE Trans. Biomed. Eng. BME-31, 633-644 (1984).

10. A. M. Clarke, W. J. Geeraets, and W. T. Ham Jr., "An equilibrium thermal model for retinal injury from optical sources," Appl. Opt. 8(5), 1051-1054 (1969). 